\title{
Predição de Valores Genéticos para a Produção de Leite no Dia do Controle e para a Produção Acumulada até 305 Dias ${ }^{1}$
}

\author{
Lenira El Faro 2,3 , Lucia Galvão de Albuquerque 4,5
}

\begin{abstract}
RESUMO - Foram estimados valores genéticos para as produções de leite no dia do controle de animais da raça Caracu, usando modelo unicaracterístico padrão (TDMO) e modelo de regressão aleatória (MRA). Os animais foram classificados com base na produção acumulada até 305 (PTA305), como tradicionalmente é feito em gado de leite. Além das produções na semana do controle, o MRA proporcionou predições de valores genéticos para produção até 305 dias, obtidos pela soma dos valores genéticos diários (MRA305). Foram estimadas as correlações de ordem (spearman) entre os valores genéticos estimados pelas diferentes metodologias, observando-se coincidência de touros classificados como deca 1 (10\% melhores touros). As curvas de lactação genéticas para os cinco melhores touros foram comparadas. As correlações de ordem entre os valores genéticos para as produções de leite na semana do controle, preditos do TDMO foram menores que 0,80. Para o MRA as correlações de ordem foram maiores, variando de 0,41 a 1,00. Entre PTA305 e MRA305 a correlação de ordem foi de 0,87 para touros. Esse valor indica que, apesar de alta, essa correlação não assegura coincidência de classificação para os touros. Verificou-se também que os melhores touros para PTA305 não apresentaram as melhores curvas de lactação genéticas estimadas pelo MRA. O MRA parece ser mais adequado que o TDMO para substituir a produção acumulada até 305 dias em programas de avaliação genética.
\end{abstract}

Palavras-chave: avaliação genética, função de covariância, raça Caracu, regressão aleatória, produção de leite

\section{Predicting Breeding Values for Test-day Records and Accumulated 305 day Milk Yield}

\begin{abstract}
Breeding values were predicted for test day milk yields of Caracu cows, using univariate model (TDMO) and random regression model (RRM). The ranks were based on traditional 305-day milk yield breeding values (PTA305). For RRM, breeding values were predicted for individual test-day milk yield and for sum of all test-days (RRM305). Spearman correlation were estimated among predicted breeding values from different methodos (TDMO and RRM) and the coincidence of rank of $10 \%$ best sires were verified. Genetic lactation curves of five best sires were compared. Spearman correlation between breeding values predicted for test-day milk yield by TDMO were lower than 0.80 . When predicted by RRM, the correlations between breeding values were higher than by TDMO, ranging from 0.41 to 1.00 . This correlation was 0.87 between PTA305 and MRA305. It was also observed that the best sires for PTA305 did not present the best genetic lactation curves, estimated by RRM. The RRM shows more adequacy than TDMO to substitute traditional 305-day milk yield in genetic evaluation of dairy cattle.
\end{abstract}

Key Words: genetic evaluation, Caracu breed, covariance function, random regression, milk yield

\section{Introdução}

A seleção para produção de leite em bovinos leiteiros baseia-se, tradicionalmente, no mérito genético para a produção acumulada até 305 dias (P305). O cálculo das produções é feito por meio da somatória das aproximações diárias, posto que as medidas são mensais, e de fatores de extensão, para lactações em andamento.

Além da produção aos 305 dias, características como a persistência da lactação têm sido objeto de vários estudos, graças a sua importância econômica, mas não fazem parte de programas de melhoramento genético. Estudos envolvendo modelagem e descrição da curva de lactação e seus componentes são amplamente discutidos na literatura. Entretanto, inexistem programas de seleção que visem progressos genéticos para a forma da curva, dada a incerteza de se obter bons ajustes utilizando-se funções matemáticas na modelagem de curvas individuais, a interpretação dos parâmetros e das funções dos parâmetros estimados para cada função, além de não se considerar a estrutura de parentesco entre os animais na estimação das curvas de cada animal.

\footnotetext{
1 Parte da tese de doutorado do primeiro autor, financiada pelo CNPq.

${ }^{2}$ Aluno de doutorado - FCAV/UNESP, Rod. Paulo D. Castelani km 8, CEP 14870-000, Jaboticabal-SP.

3 Pesquisador do Instituto de Zootecnia, APTA/SAA/SP. (Ienira@aptaregional.sp.gov.br)

${ }^{4}$ Prof. Adjunto - FCAV/UNESP, Jaboticabal-SP. (Igalb@fcav.unesp.br)

5 Pesquisador do CNPq.
} 
As metodologias propostas, atualmente, para a avaliação genética buscam minimizar alguns problemas quando da utilização da P305. Elas permitem estimar com maior acurácia os fatores ambientais que agem sobre a produção de leite em períodos parciais da lactação; incluir nas avaliações genéticas animais cujas lactações estão em andamento, sem utilizar fatores para a extensão da produção; incluir nas avaliações genéticas animais cujas lactações têm perda de controles leiteiros intermediários; utilizar os dados observados, e não os estimados nas avaliações; e aumentar o número de informações e, principalmente, a acurácia na avaliação de animais jovens.

Essas metodologias - denominadas "Test-Day Models" ou "Modelos para o Dia do Controle" - têm sido rapidamente difundidas em melhoramento genético, como alternativas presentes e futuras para avaliar a produção de leite. As abordagens dos TDM são feitas sob diferentes aspectos metodológicos, que podem ser classificados como TDM ordinários (TDMO), fixos ou de dimensão finita e de dimensão infinita, conhecidos como Modelos de Regressão Aleatória (MRA). Os TDMO são aqueles em que as produções no dia do controle são analisadas como características distintas, consideradas em análises unicaracterísticas ou multicaracterísticas. Esses modelos consideram variações adicionais referentes ao dia do controle, como grupo de manejo, data do controle, número de ordenhas, rebanho, enre outros (Gadini, 1997; Van Der Werf \& Schaeffer, 1997; Meyer, 1998b).

Os TDMO mais simples são os modelos de repetibilidade, que assumem estrutura univariada, variâncias homogêneas entre todos os controles e correlações genéticas entre si iguais à unidade, o que, na realidade, não ocorre. Observa-se que as correlações genéticas entre os controles são elevadas, sobretudo entre os adjacentes, mas não iguais a um. Entre os problemas relacionados ao uso do TDMO, destacamse o elevado número de características a serem analisadas, o tempo computacional necessário para estimação dos componentes de (co)variância, a dificuldade por parte do criador em se utilizar os resultados, a determinação dos critérios de seleção, baseando-se em um ou mais controles, e a desconsideração da forma da curva de lactação dos animais e seu principal componente - a persistência da lactação (Schaeffer, 1996; Jamrozik \& Schaeffer, 1997).

Os Modelos de Regressão Aleatória (MRA) apresentam as mesmas vantagens dos TDMO, além de considerar a forma da curva de lactação e de analisar os $n$ controles por meio de uma estrutura unicaracterística. Também permitem identificar a curva de lactação genética para cada animal, como desvios da trajetória populacional ou de grupos de indivíduos, e podem, no futuro, permitir a seleção de animais cujas curvas sejam mais persistentes (Ptak \& Schaeffer, 1993; Schaeffer, 1996; Jamrozik \& Schaeffer, 1997).

A definição de critérios de seleção mediante a aplicação dos MRA ou dos TDMO padrão tem relevante importância em programas de melhoramento genético.

Foram estimados os valores genéticos dos animais para as produções de leite no dia do controle e para a produção até 305 dias de lactação (P305) e comparada a classificação dos animais, quando estas estimativas foram obtidas mediante modelo de regressão aleatória (MRA), e análises unicaracterísticas padrão ou modelo usando a produção de leite no dia do controle (TDMO).

\section{Material e Métodos}

Neste estudo, foram utilizados 86.598 controles leiteiros semanais de 2.155 primeiras lactações de vacas Caracu pertencentes ao rebanho da fazenda Chiqueirão, Poços de Caldas, MG. As lactações com duração superior a 305 dias foram truncadas neste período, empregando-se aqueles que apresentaram de 6 até 43 controles leiteiros, sendo que mais de $94 \%$ contiveram 35 ou mais controles. A matriz de parentesco conteve 2.706 animais (189 eram machos).

As produções de leite no dia do controle foram analisadas por análises padrão, unicaracterísticas (TDMO) ou Regressão Aleatória (MRA). A lactação foi dividida em 43 classes semanais, uma vez que o sistema de controle leiteiro adotado pela propriedade foi o semanal. Para o TDMO foram usadas apenas 21 classes semanais $(2,4,6, \ldots 42$ semanas $)$, ao passo que para o MRA, todas as 43 classes de controle semanais. As produções de leite foram, dessa maneira, as mesmas para ambos os modelos.

\section{Análise unicaracterística (TDMO)}

Para essa análise foi usado o modelo animal, considerando-se, separadamente, as 21 produções de leite no dia do controle. Neste modelo, foram considerados os efeitos fixos de grupo contemporâneo, composto pelas variáveis ano e mês do controle e retiro, e a idade 
da vaca ao parto como covariável (efeitos linear e quadrático). Como aleatórios, foram considerados os efeitos de animal e temporário de ambiente.

\section{Modelo de Regressão Aleatória (MRA)}

Para o MRA foram considerados os mesmos efeitos fixos e aleatórios anteriormente citados, incluindo a trajetória média da população (covariáveis dia em lactação sobre a produção de leite semanal) e o efeito aleatório permanente de ambiente. Os componentes de variância para os efeitos aleatórios genético aditivo e permanente de ambiente foram modelados por meio de funções de covariância, usando polinômios ortogonais de Legendre de quinta ordem. O grupo contemporâneo apresentou 1.582 níveis e foi definido pelas variáveis retiro, ano e mês do controle. A trajetória média da população (fixa) foi modelada por um polinômio de Legendre de terceira ordem. Os resíduos foram considerados independentemente distribuídos, com variâncias heterogêneas, modeladas usando-se função de variâncias (FV) ajustada com um polinômio ordinário de sexta ordem.

As ordens das funções de covariância genéticoaditivas e permanentes de ambiente, bem como a estrutura de variâncias residuais adotada no presente estudo foram definidas após análises, testando alguns modelos alternativos (El Faro, 2002).

O modelo de regressão aleatória geral pode ser representado por:

$$
y_{i j}=F+\sum_{m=0}^{k_{b}-1} \beta_{m} \phi_{m}\left(t_{i}\right)+\sum_{m=0}^{k_{a}-1} \alpha_{j m} \phi_{m}\left(t_{i j}\right)+\sum_{m=0}^{k_{a p}-1} \gamma_{j m} \phi_{m}\left(t_{i j}\right)+\varepsilon_{i j}
$$

em que: $y_{i j}=$ produção de leite da i-ésima classe de semana de lactação, pertencente ao j-ésimo animal (i=1, $2, \ldots ., 43) ; F=$ conjunto de efeitos fixos, exceto a curva de lactação média populacional; $\beta_{m}=$ conjunto de $\mathrm{m}$ regressores fixos para a curva média da população; $\phi_{m}\left(t_{i}\right)=$ função de regressão de ordem $\mathrm{k}_{\mathrm{b}}$ que descreve a curva média da população de acordo com a semana de lactação $\left(\mathrm{t}_{\mathrm{j}}\right) ; \phi_{m}\left(t_{i j}\right)=$ funções de regressão que descrevem as trajetórias de cada indivíduo j, de acordo com a semana de lactação $\left(\mathrm{t}_{\mathrm{j}}\right)$, para os efeitos aleatórios genético-aditivos e permanentes de ambiente; $\alpha_{j m}, \gamma_{j m}=m$ regressores aleatórios genéticoaditivos e permanentes de ambiente, para cada animal $\mathrm{j}$; $k_{b}, k_{a} e k_{a p}=$ ordens dos polinômios ortogonais utilizados para descrever a curva média e os efeitos genéticoaditivos e permanentes de ambiente, respectivamente, em que $k_{b}=4, k_{a}$ e $k_{a p}=6 ; \varepsilon_{i j}=$ erro aleatório associado a cada controle i do animal j.
Na forma matricial, o modelo pode ser representado por:

$$
\mathrm{y}=\mathrm{Xb}+\mathrm{Za}+\mathrm{Wap}+\mathrm{e}
$$

em que: $\mathrm{y}=$ vetor das $\mathrm{N}$ observações, medidas em $\mathrm{N}_{\mathrm{d}}$ animais com registros; $b=0$ vetor de efeitos fixos que inclui as soluções para GC e para as covariáveis idade ao parto e semana de lactação; a = vetor de soluções para os coeficientes de regressão aleatórios genéticoaditivos; ap = vetor de soluções para os coeficientes de regressão aleatórios do efeito permanente de ambiente; $\mathrm{X}, \mathrm{Z}, \mathrm{W}=$ matrizes de incidência para os efeitos fixos, aleatórios genético-aditivos e permanentes de ambiente, respectivamente; e e $=$ o vetor dos resíduos;

A dimensão do vetor a é de $\mathrm{k}_{\mathrm{a}} \mathrm{xN}_{\mathrm{a}}$ coeficientes, sendo que $\mathrm{k}_{\mathrm{a}}$ representa o número de parâmetros da função de regressão e $\mathrm{N}_{\mathrm{a}}$, o número de animais no arquivo de dados. $\mathrm{O}$ vetor ap tem dimensão de $\mathrm{k}_{\mathrm{ap}} \mathrm{xN}_{\mathrm{d}}$ coeficientes, sendo que $\mathrm{k}_{\mathrm{ap}}$ representa o número de parâmetros da função de regressão para ambiente permanente e $\mathrm{N}_{\mathrm{d}}$, o números de animais com registros.

As pressuposições em relação aos componentes do modelo são:

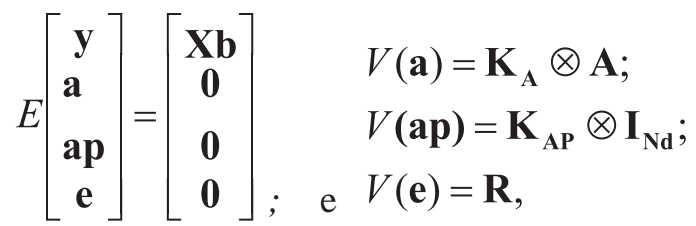

em que: $K_{A}$ e $K_{A P}$ são as matrizes de coeficientes para as funções de covariância cujos elementos representam as (co)variâncias entre os coeficientes de regressão aleatórios genético-aditivos e permanentes de ambiente, respectivamente; $\mathbf{A}$, a matriz de coeficientes de parentesco entre os animais; $\mathrm{I}_{\mathrm{Nd}}$, uma matriz

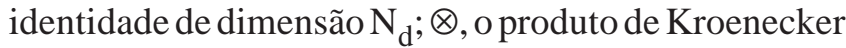
ou produto direto entre matrizes; $\mathrm{R}$, uma matriz diagonal contendo as variâncias residuais.

Além das produções na semana do controle analisadas pelas duas metodologias (TDMO e MRA), analisou-se a produção de leite acumulada até 305 dias (P305), por meio de modelo animal que considerou o efeito fixo de grupo contemporâneo, composto pelas variáveis ano e mês do parto, e a idade da vaca ao parto como covariável (efeitos linear e quadrático). Como efeitos aleatórios, foram considerados os efeitos de animal e temporários de ambiente.

Os componentes de variância foram estimados pelo método da Máxima Verossimilhança Restrita, 
usando um algoritmo livre de derivadas, disponível no pacote MTDFREML (Boldman et al., 1993) para o TDMO e a P305. Para a regressão aleatória, foi usada a opção DXMRR do pacote estatístico DFREML (Meyer, 1998a).

Os valores genéticos para as produções de leite na semana do controle foram preditos para as duas metodologias aplicadas (TDMO e MRA). A classificação dos animais foi comparada com base na classificação para a produção acumulada até 305 dias (P305), pelo método tradicional. Adicionalmente, foram preditos os valores genéticos para a produção acumulada até 305 dias pelo MRA, obtidos pela somatória dos valores genéticos preditos em cada dia da lactação, uma vez que cada animal apresentou um conjunto de seis valores genéticos referentes aos coeficientes de regressão aleatórios. O cálculo dos valores genéticos diários para cada animal (VGij) baseou-se na seguinte equação:

$$
\begin{aligned}
\mathrm{VG}_{(\mathrm{ij})}= & \mathrm{VG}_{0(\mathrm{j})}+\mathrm{VG}_{1(\mathrm{j})} \mathrm{X}_{(\mathrm{i})}+\mathrm{VG}_{2(\mathrm{j})} \mathrm{X}_{(\mathrm{i})}^{2}+\mathrm{VG}_{3(\mathrm{j})} \\
& \mathrm{X}_{(\mathrm{i})}^{3}+\mathrm{VG}_{4(\mathrm{j})} \mathrm{X}_{(\mathrm{i})}^{4}+\mathrm{VG}_{5(\mathrm{j})} \mathrm{X}_{(\mathrm{i})}^{5}
\end{aligned}
$$

em que: $V_{0(j)}$ a $V G_{5(j)}$ são os valores genéticos do intercepto até o coeficiente de quinta ordem, dada a ordem da função de covariâncias usada para descrever o efeito genético aditivo, pertencentes ao j-ésimo animal, e $\mathrm{X}_{(\mathrm{i})}$, o i-ésimo dia em lactação padronizado para o intervalo -1 a 1.

A padronização dos dias em lactação foi feita de acordo com a fórmula citada por Kirkpatrick et al. (1990):

$$
\begin{gathered}
\mathrm{X}_{(\mathrm{i})}=\left[2\left(\mathrm{DEL}_{(\mathrm{i})}-\mathrm{DEL}_{(\text {min })}\right) /\left(\mathrm{DEL}_{(\text {max })}-\right.\right. \\
\left.\left.\operatorname{DEL}_{(\text {min })}\right)\right]-1
\end{gathered}
$$

em que: $\operatorname{DEL}_{(i)}$ é o i-ésimo dia em lactação; $\mathrm{DEL}_{(\text {min) }}$, o menor dia em lactação; e $\mathrm{DEL}_{(\text {max })}$ o maior dia em lactação.

A comparação dos animais coincidentes foi realizada pela correlação de ordem, usando o procedimento Spearman (SAS, 1992), para os 2.706 animais e para diferentes proporções dos 189 touros selecionados.

\section{Resultados e Discussão}

As variâncias fenotípicas $\left(\sigma_{p}^{2}\right)$ e genéticas $\left(\sigma_{a}^{2}\right)$ estimadas para a produção de leite na semana de lactação para o modelo de regressão aleatória, juntamente com as estimadas para o TDMO, são apresentadas na Figura 1. As $\sigma_{p}^{2}$ estimadas pelo MRA foram muito próximas das estimadas pelas análises unicaracterísticas. Poucas diferenças ocorreram para as $\sigma^{2}$ a estimadas pelo TDMO e MRA. No geral, tanto para os MRA quanto para TDMO, as variâncias foram maiores no início da lactação, diminuindo nas demais fases até o final, exceto a variância genética, que mostrou leve tendência de aumento no final da lactação.

A estimativa de $\mathrm{h}^{2}$ para a produção acumulada até 305 dias (P305) foi de 0,27. Para o MRA e o TDMO, as estimativas estão apresentadas na Figura 2. As $h^{2}$ estimadas pelo TDMO variaram entre $0,09\left(12^{\mathrm{a}}\right.$ semana) e 0,32 ( $42^{\mathrm{a}}$ semana), com oscilações nos valores no decorrer da lactação. As maiores $\mathrm{h}^{2}$ ocorreram na $28^{\mathrm{a}}$, $32^{\mathrm{a}}, 40^{\mathrm{a}}$ e $42^{\mathrm{a}}$ semanas, de $0,24,0,22,0,22$ e 0,32 , respectivamente. As $\mathrm{h}^{2}$ estimadas pelo MRA variaram de 0,09 a 0,26. Em geral, as estimativas foram menores no meio da lactação, entre as semanas 14 e 30 .

Em comparação ao TDMO, as $\mathrm{h}^{2}$ estimadas para as produções de leite pelo MRA acompanharam os valores do início da lactação e foram menores no meio $\left(14^{\mathrm{a}}\right.$ a $28^{\mathrm{a}}$ semana) e nos últimos controles $\left(40^{\mathrm{a}}\right.$ a $42^{\mathrm{a}}$ semana) da lactação. Os valores do início da lactação $\left(1^{\mathrm{a}}\right.$ a $13^{\mathrm{a}}$ semana) foram levemente maiores para o MRA que para o TDMO.

As estimativas de $\mathrm{h}^{2}$ obtidas no presente estudo seguiram a mesma tendência observada na literatura, com maiores herdabilidades no início e no final da lactação (Strabel \& Misztal, 1999; Tijani et al., 1999; Brotherstone et al., 2000). Entretanto, em magnitude, as $\mathrm{h}^{2}$ estimadas neste estudo foram menores que as relatadas por Jamrozik \& Schaeffer (1997), Jamrozik et al. (1997a,b) e Olori (1999a,b), devido à maneira como modelaram o efeito permanente de ambiente, considerando-o uma constante por todo o período da lactação, o que pode ter inflacionado as variâncias genéticas.

Na Tabela 1, são apresentadas algumas estatísticas simples dos valores genéticos preditos para as produções na semana do controle, usando TDMO (tdm2-tdm42) e MRA (mra2-mra42), e para a P305. Para o MRA, os valores genéticos para as produções foram estimados usando-se as soluções obtidas para os coeficientes de regressão aleatória para cada animal incluído na matriz de parentesco. $=$ Os valores genéticos preditos para as produções de leite na semana do controle,bem como a amplitude desses valores genéticos, foram maiores pelo MRA que para 

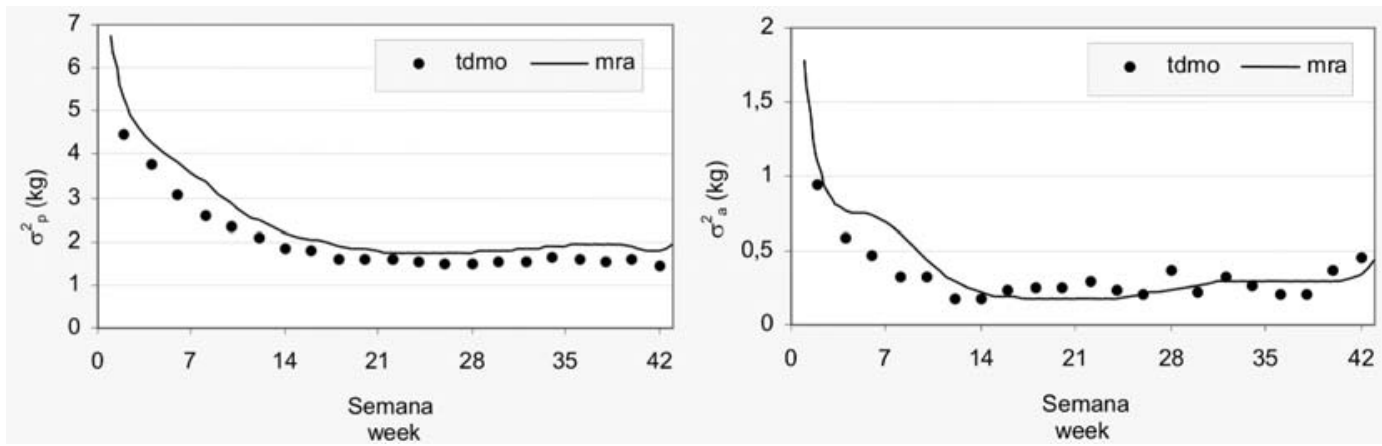

Figura 1 - Variâncias fenotípicas $\left(\sigma^{2}\right.$ p $)$ e genéticas $\left(\sigma^{2}{ }_{a}\right)$ estimadas para as produções na semana de lactação pelo modelo unicaracterístico (TDMO) e modelo de regressão aleatória (MRA).

Figure 1 - Phenotypic $\left(\sigma_{p}^{2}\right)$ and genetic $\left(\sigma^{2}{ }_{a}\right)$ variances estimated for test-day milk yield by ordinary testday model (TDMO) and by random regression model (MRA).

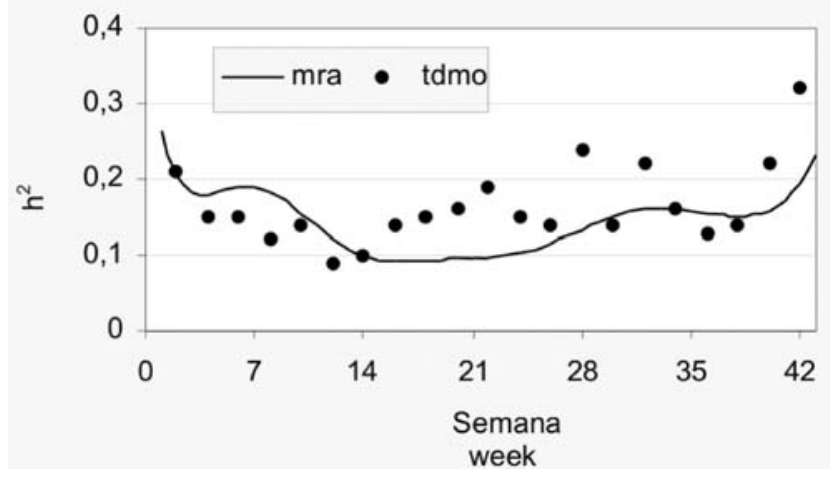

Figura 2 - Estimativas de $\mathrm{h}^{2}$ para as produções de leite no dia do controle para TDMO e MRA.

Figure 2 - Heritability estimates for test-day milk yield by ordinary test-day model (TDMO) and by random regression model (MRA).

o TDMO. O mesmo resultado foi constatado para os valores genéticos preditos para a produção acumulada até 305 dias pelo MRA (MRA305) e pelo método tradicional (PTA305).

As correlações de ordem entre os valores genéticos preditos para as produções semanais pelo TDMO variaram de 0,13 a 0,70 para touros e de 0,13 a 0,64 para todos os animais (Figura 3). Pela superfície de resposta, observa-se que as correlações são maiores entre produções mais próximas, principalmente entre produções do meio da lactação, reduzindo com o aumento da distância entre elas. Para o MRA (Figura 4), as correlações foram bem maiores, variando de 0,46 a 1,00 entre os valores genéticos de touros e de 0,41 a 1,00 entre os valores genéticos de todos os animais, sendo que a maior parte variou de 0,80 a 1,00. Estes resultados indicam que pode haver maior coincidência de animais classificados para as produções no dia do controle, quando seus valores genéticos foram preditos usando regressão aleatória.

A diferença na magnitude das correlações de ordem entre os valores genéticos pode ser explicada, principalmente, pela abordagem de cada metodologia na modelagem das produções em cada controle. $\mathrm{O}$ TDMO considera cada característica separadamente em análises com uma única ou mais características, diferentemente da abordagem feita pela regressão aleatória, em que as matrizes de incidência são estruturadas, obedecendo uma ordem na estimação dos componentes de (co)variância para cada fase da

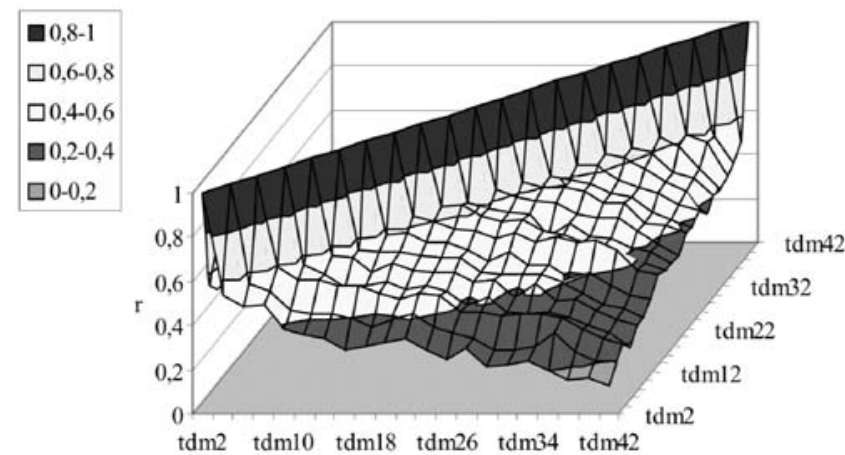

Figura 3 - Correlações de ordem (r) entre os valores genéticos de todos os animais, estimados para as produções de leite em cada semana de lactação (TDMO).

Figure 3 - Rank correlation (r) between predicted breeding values for test-day milk yield by unitrait analysis (ordinary tes-day model). 
Tabela 1 - Média, desvio-padrão (DP) e amplitude dos valores genéticos preditos (VGE) para a produção até 305 dias e para as produções de leite no dia do controle de acordo com o modelo de regressão aleatória e com o test-day model

Table 1 - Means, standard deviation and range of breeding values predicted (EBV) for 305-day milk yield and for test-day milk yields by random regression model and by test-day model

\begin{tabular}{|c|c|c|c|c|c|c|c|c|c|}
\hline \multicolumn{5}{|c|}{$\begin{array}{l}\text { Regressão aleatória } \\
\text { Random regression }\end{array}$} & \multicolumn{5}{|c|}{ Test-day model } \\
\hline VGE & média & DP (kg) & mínimo & máximo & VGE & média & DP (kg) & mínimo & máximo \\
\hline$E B V$ & mean & $S D$ & minimum & maximum & $E B V$ & mean & $S D$ & minimum & maximum \\
\hline mra2 & $-0,017$ & 0,598 & $-1,813$ & 2,685 & $t d m 2$ & 0,035 & 0,475 & $-1,555$ & 2,159 \\
\hline mra4 & 0,037 & 0,551 & $-1,513$ & 2,551 & $t d m 4$ & 0,015 & 0,328 & $-0,964$ & 1,302 \\
\hline mra6 & 0,074 & 0,517 & $-1,399$ & 2,415 & $\operatorname{tdm} 6$ & 0,02 & 0,301 & $-1,167$ & 1,258 \\
\hline mra8 & 0,099 & 0,49 & $-1,304$ & 2,28 & $\operatorname{tdm} 8$ & 0,018 & 0,224 & $-0,645$ & 1,088 \\
\hline mra10 & 0,114 & 0,467 & $-1,225$ & 2,15 & $\operatorname{tdm} 10$ & 0,036 & 0,235 & $-0,646$ & 0,853 \\
\hline mra12 & 0,122 & 0,448 & $-1,159$ & 2,027 & $\operatorname{tdm} 12$ & 0,013 & 0,146 & $-0,654$ & 0,51 \\
\hline mra14 & 0,126 & 0,43 & $-1,108$ & 1,922 & $\operatorname{tdm} 14$ & 0,011 & 0,146 & $-0,53$ & 0,672 \\
\hline mra16 & 0,127 & 0,416 & $-1,081$ & 1,845 & $\operatorname{tdm} 16$ & 0,003 & 0,199 & $-0,766$ & 0,834 \\
\hline $\operatorname{mra} 18$ & 0,126 & 0,403 & $-1,067$ & 1,773 & $\operatorname{tdm} 18$ & 0,006 & 0,207 & $-0,71$ & 0,849 \\
\hline $\operatorname{mra} 20$ & 0,125 & 0,393 & $-1,079$ & 1,709 & $\operatorname{tdm} 20$ & 0,011 & 0,213 & $-0,787$ & 0,775 \\
\hline $\operatorname{mra} 22$ & 0,124 & 0,384 & $-1,092$ & 1,652 & $\operatorname{tdm} 22$ & 0,011 & 0,246 & $-0,811$ & 0,921 \\
\hline $\operatorname{mra} 24$ & 0,123 & 0,378 & $-1,106$ & 1,601 & $\operatorname{tdm} 24$ & 0,022 & 0,21 & $-0,648$ & 0,785 \\
\hline $\operatorname{mra} 26$ & 0,122 & 0,373 & $-1,121$ & 1,616 & $\operatorname{tdm} 26$ & 0,011 & 0,196 & $-0,623$ & 0,664 \\
\hline $\operatorname{mra} 28$ & 0,121 & 0,37 & $-1,137$ & 1,638 & $\operatorname{tdm} 28$ & 0,009 & 0,306 & $-1,076$ & 1,23 \\
\hline mra30 & 0,12 & 0,368 & $-1,152$ & 1,662 & $\operatorname{tdm} 30$ & 0,007 & 0,197 & $-0,605$ & 0,826 \\
\hline mra32 & 0,118 & 0,366 & $-1,165$ & 1,686 & $\operatorname{tdm} 32$ & 0,024 & 0,276 & $-1,137$ & 1,051 \\
\hline mra34 & 0,118 & 0,366 & $-1,177$ & 1,712 & $\operatorname{tdm} 34$ & 0,008 & 0,221 & $-0,956$ & 0,822 \\
\hline mra36 & 0,117 & 0,367 & $-1,184$ & 1,74 & $\operatorname{tdm} 36$ & 0,007 & 0,175 & $-0,708$ & 0,732 \\
\hline mra38 & 0,117 & 0,368 & $-1,188$ & 1,771 & $\operatorname{tdm} 38$ & 0,007 & 0,175 & $-0,734$ & 0,974 \\
\hline mra40 & 0,12 & 0,372 & $-1,186$ & 1,808 & $\operatorname{tdm} 40$ & 0,02 & 0,261 & $-0,971$ & 1,543 \\
\hline mra42 & 0,125 & 0,378 & $-1,177$ & 1,853 & $\operatorname{tdm} 42$ & 0,025 & 0,302 & $-1,161$ & 1,338 \\
\hline MRA $305^{1}$ & 32,215 & 119,157 & $-332,194$ & 521,209 & PTA $305^{2}$ & 6,584 & 86,387 & $-240,911$ & 331,526 \\
\hline
\end{tabular}

lactação. Espera-se maior proximidade entre os resultados, caso as análises para o TDMO fossem feitas considerando-se as 21 características conjuntamente, em razão do elevado número de características, da exigência computacional e da dificuldade de se alcançar a convergência.

Todas as correlações de ordem entre os valores genéticos preditos para as produções em cada semana pelo MRA e pelo TDMO apresentaram valor abaixo de $80 \%$, quando foram considerados os valores genéticos preditos para os touros ou para todos os animais. Mesmo entre semanas idênticas, por exemplo, mra2 com tdm2, mra4 com tdm4, e assim por diante (Figura 5), essas correlações foram, no máximo, de 0,74 para touros e 0,70 para todos os animais.

As correlações de ordem entre as produções acumuladas até 305 dias pelo método tradicional (PTA305) e pelo modelo de regressão aleatória, em

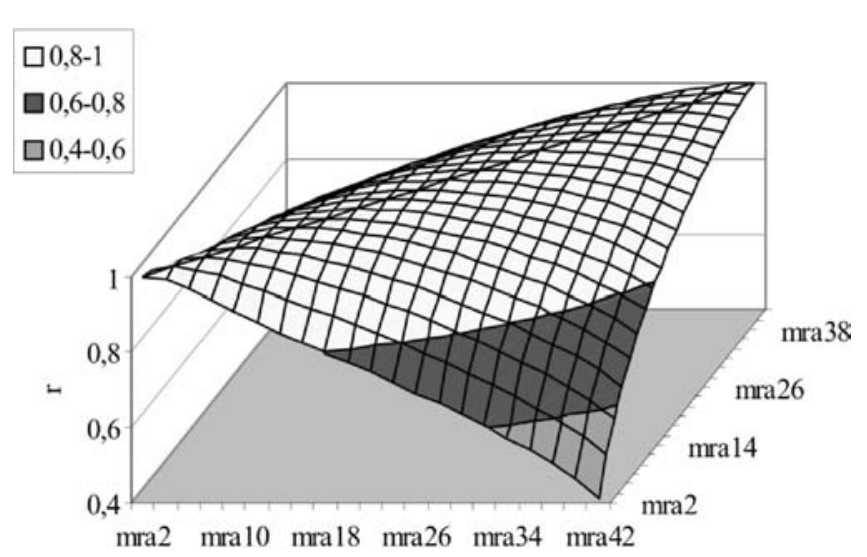

Figura 4 - Correlações de ordem (r) entre os valores genéticos de todos os animais, estimados para as produções de leite em cada semana de lactação pelo MRA.

Figure 4 - Rank correlation ( $r$ ) between predicted breeding values for test-day milk yields by random regression model. 
que foram somados os valores genéticos estimados em cada dia da lactação (MRA305), foram de 0,85 e 0,88 , respectivamente, para todos os animais e para machos. Apesar de elevados, esse valores indicam que podem ocorrer divergências na classificação dos animais para as duas abordagens.

Na Figura 6, é possível observar que as maiores correlações de ordem estimadas entre os valores genéticos preditos para P305 (PTA305) e para as produções de leite em cada semana foram maiores pelo MRA que pelo TDMO. A maioria das correlações foi superior a 0,80 entre PTA305 e os valores genéticos pelo MRA, enquanto entre PTA305 e o TDMO não atingiu 0,80 em nenhum estádio da lactação. Essas correlações foram ainda maiores entre os valores genéticos para as produções em cada semana e MRA305. As produções do meio da lactação apresentaram as maiores correlações de Spearman com a P305, sendo que alguns valores foram iguais a um (mra16 a mra24 com MRA305). Para o TDMO as correlações de ordem entre as produções em cada semana e as produções acumuladas até 305 apresentaram valores muito próximos, independentemente da maneira como foi predito o valor genético para a P305 (PTA305 ou MRA305).

Os resultados representados na Figura 6 podem indicar coincidência de animais superiores com base na P305 e também superiores para a curva de lactação genética, obtida pelo MRA. É importante averiguar a maneira como ocorre essa coincidência e sua intensidade, pois apenas a correlação de ordem não garante a seleção dos mesmos animais, usando-se como critério a PTA305 ou a MRA305. Além disso, há a necessidade de identificar, para esse rebanho, animais que transmitam aos seus descendentes curvas de lactação com formas mais desejáveis.
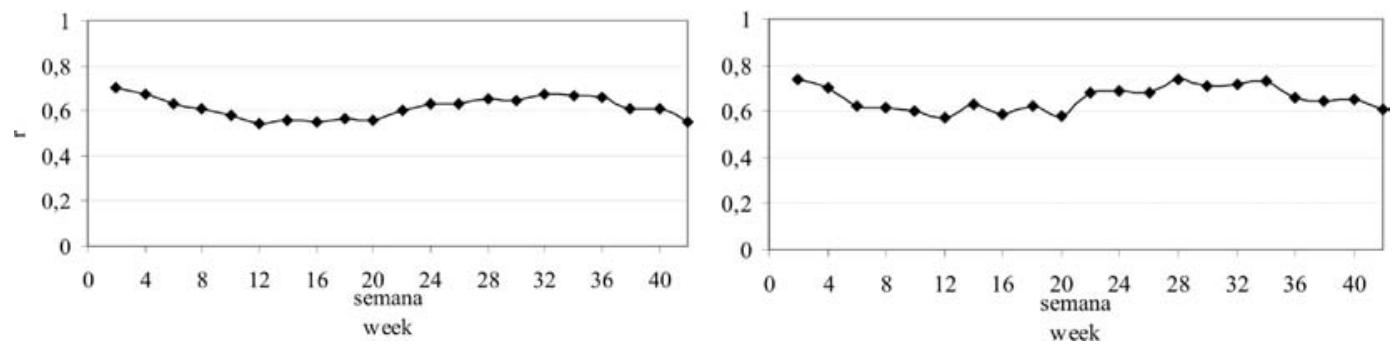

Figura 5 - Correlações de ordem (r) entre os valores genéticos preditos para produção de leite em semanas idênticas, por métodos diferentes (Regressão aleatória e test-day model), para todos os animais do rebanho (à esquerda) e para os machos (à direita).

Figure 5 - Rank correlation (r) between predicted breeding values for test-day milk yield in identical classes, by distinct methods (random regression and ordinary test-day model), for all males (right) and all animals (left).
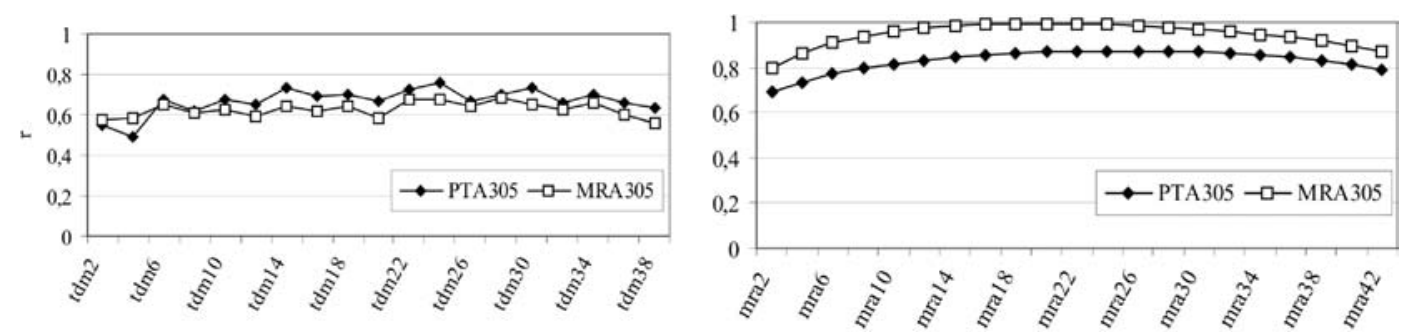

Figura 6 - Correlações de ordem $(r)$ entre os valores genéticos preditos para a produção acumulada até 305 dias, pelo método tradicional (PTA305) e por Regressão aleatória (MRA305), e as produções de leite em cada semana de lactação, pelo TDMO (à esquerda) e pelo MRA (à direita).

Figure 6 - Rank correlation (r) between predicted breeding values for 305-day milk yield, by unitrait analysis (PTA305) and by random regression (MRA305) and test-day milk yields using test-day model (left) and random regression (right). 
Na Tabela 2, são apresentadas as classificações dos $10 \%$ melhores touros (deca 1), com base no valor genético predito para P305 (PTA305) e suas respectivas classificações para MRA305 e para as produções de leite nas semanas de lactação pelo TDMO e pelo MRA. Em relação à PTA305 e MRA305, a correlação de ordem entre os valores genéticos preditos para todos os touros reduziu de 0,87 para 0,32 , quando apenas os $10 \%$ melhores touros foram selecionados para PTA305. Entretanto, apesar da não coincidência na classificação (rank) dos touros, há certa coincidência de decas, principalmente para os 19 touros deca 1. Entre os 19 touros classificados como deca 1 para PTA305, 14 também foram classificados para MRA305. Nas decas restantes, esse número diminuiu. Por exemplo, para as decas $2,3,4,5,6,7,8,9 \mathrm{e} 10$, coincidiram, respectivamente, $6,6,6,3,3,7,6,3$ e 9 touros.

Em relação às produções de leite nas semanas de lactação, observa-se que os melhores touros segundo a classificação para PTA305 não foram os melhores de acordo com o TDMO e MRA. Para o TDMO, houve grande disparidade nas classificações em cada semana, não indicando tendência e dificultando a escolha de um animal. Para o MRA, entretanto, observaram-se disparidades, que, entretanto, foram menores. Quando a classificação dos $10 \%$ melhores touros baseou-se no valor genético para MRA305 (Tabela 3), observou-se maior coincidência de ordem entre MRA305 e os valores genéticos preditos para as semanas pelo MRA, o que era esperado, em decorrência do modo como a metodologia considera as produções em cada controle e da maneira como foi estimado o valor genético para a produção acumulada (MRA305).

As curvas de lactação (genéticas) dos cinco melhores touros classificados de acordo com os valores genéticos para PTA305 (Figuras 7 e 8) ajudam a ressaltar as diferenças entre as duas metodologias (TDMO e MRA). Para o MRA são estimadas curvas de lactação genéticas, que são desvios da curva de lactação média da população (Schaeffer, 1996), ao contrário do TDMO, que proporciona predições de valores genéticos para cada produção como características distintas. Os valores genéticos preditos para as produções em cada controle usando o TDMO foram menores que os preditos pelo MRA e oscilaram por toda a lactação. Alguns animais apresentaram valores baixos e até negativos em algumas fases da lactação, quando seus valores genéticos foram preditos usando-se TDMO. Na Figura 7, observa-se que o melhor touro classificado para PTA305 (t78036) não apresentou a curva de lactação genética com a melhor produção inicial, e nem a mais persistente; esse touro seria o $4^{\circ}$ classificado para MRA305 (Tabela 3). O segundo melhor touro para PTA305 (t89341) seria o primeiro classificado para MRA305, com uma curva genética mais persistente. É interessante observar que os touros t89341 e t84075 (respectivamente, o $2^{\circ}$ e o $28^{\circ}$ classificados para PTA305) superaram o $1^{\mathrm{o}}$ classificado, quando suas curvas foram comparadas (Figura 9), e passam a ser, respectivamente, o $1^{\circ}$ e o $2^{\circ}$ melhores touros, de acordo com MRA305. Nota-se pequenas diferenças entre suas curvas genéticas no início e no final da lactação, que comprovam a possi-

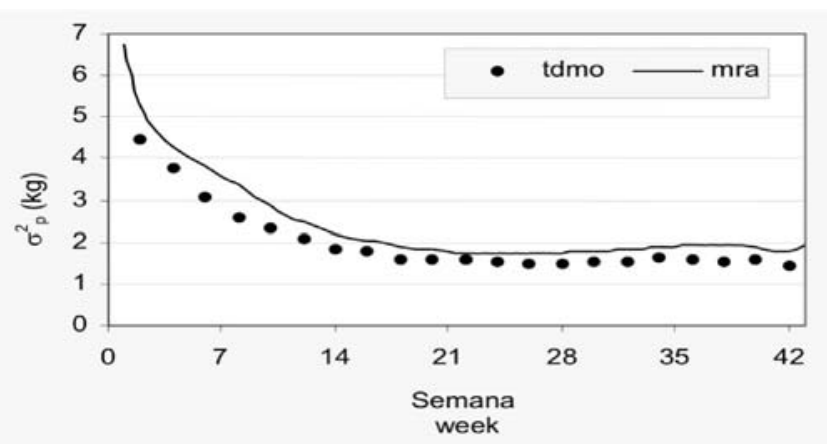

Figura 7 - Valores genéticos preditos para as produções de leite nas semanas de lactação pelo MRA, para os cinco melhores touros classificados com base na PTA305.

Figure 7 - Predicted breeding values for test-day milk yield by random regression of five best sires based on rank of PTA305.

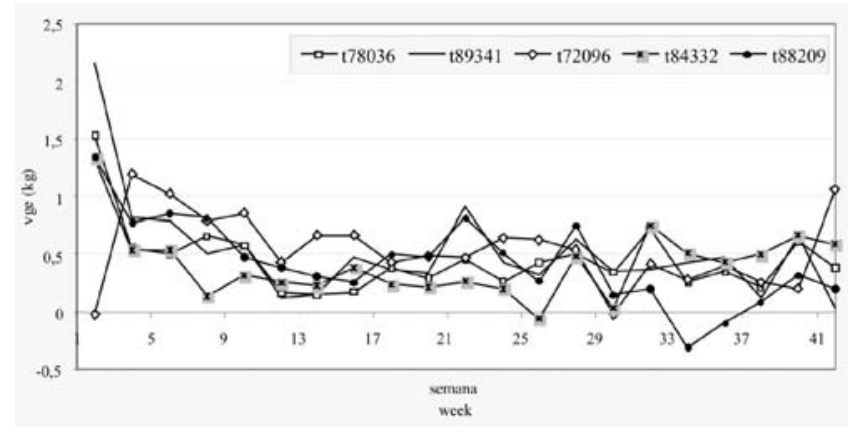

Figura 8 - Valores genéticos preditos para as produções de leite nas semanas de lactação pelo TDMO, para os cinco melhores touros classificados com base na PTA305.

Figure 8 - Predicted breeding values for test-day milk yield by TDMO of five best sires based on rank of PTA305. 


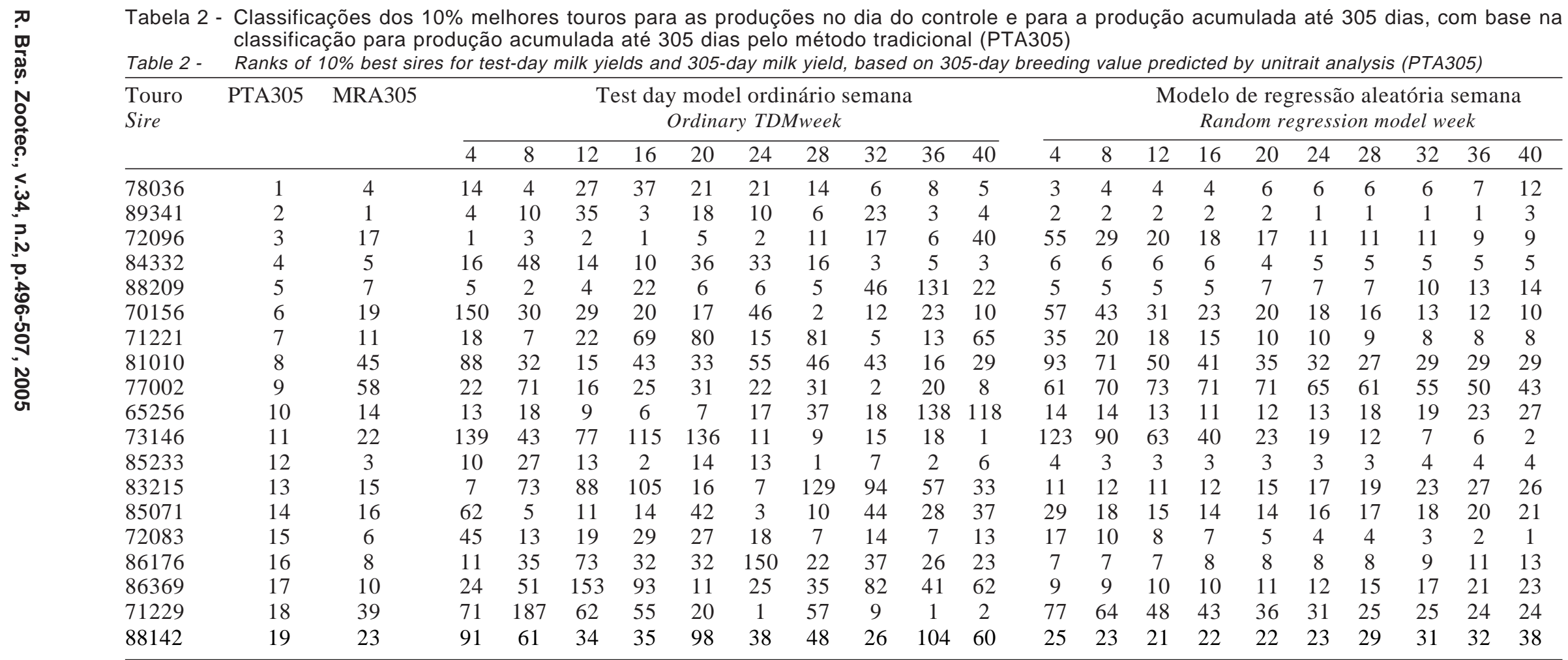


bilidade de grandes divergências ao adotar PTA305 ou MRA305 como critérios de seleção.

Os resultados encontrados para esse conjunto de dados indicam que o TDMO foi inadequado para substituir o método tradicionalmente usado para avaliação genética para a P305. Vale ressaltar que as predições dos valores genéticos proporcionadas pelo TDMO são pontuais, ou seja, são obtidas apenas nos pontos em que foram estabelecidas as classes de dia em lactação. A adoção da produção de leite em apenas um controle como critério de seleção poderia causar mudanças indesejáveis na curva de lactação (Figura 2). Um dos entraves em adotar esses modelos é o estabelecimento de critérios de seleção que proporcionem ganhos em todo o período de lactação, usando uma ou combinando várias produções ao longo do período. Outro entrave já mencionado diz respeito à abordagem metodológica do TDMO. Nesses modelos, apesar de as covariâncias entre as produções nos diversos controles variarem, não são feitas pressuposições em relação à estrutura de covariâncias, ou seja, as matrizes de (co)variâncias não são estruturadas, ao contrário dos MRA. Isso pode explicar os diferentes resultados obtidos com ambas as metodologias empregadas neste estudo.
A aplicação de resultados provenientes dos MRA em programas de melhoramento genético pode ser viável. Entretanto, a seleção baseada em fases da lactação ou em parâmetros da curva - indicativos de curvas de lactação mais persistentes - precisa ser melhor estudada. A seleção baseada em parâmetros da curva de lactação implicaria no uso de funções, cujos parâmetros tivessem interpretação biológica conhecida, que não é o caso de polinômios ortogonais. A metodologia dos MRA parece auxiliar na escolha de animais com curvas de lactação mais persistentes, podendo-se adotar como critério de seleção a produção acumulada até 305 dias (MRA305), por ser mais compreensível para os criadores familiarizados com a PTA305. A aplicação dessa metodologia poderia proporcionar ganhos econômicos para os criadores, principalmente de raças zebuínas, cujas curvas de lactação apresentam baixa persistência e lactações mais curtas. Isso seria possível por meio da escolha de touros cujas curvas de lactação tivessem desvios positivos após o pico de lactação (Figura 9), ou de fórmulas descritas na literatura para o cálculo da persistência, que se baseiam em razões de produções de leite em períodos específicos da lactação. A diferença é que, utilizandose os MRA, as razões seriam entre os valores genéticos

Tabela 3 - Classificação dos 10\% melhores touros para as produções no dia do controle, com base no valor genético para produção acumulada em até 305 dias pelo modelo de regressão aleatória (MRA305)

Table 3 - Ranks of 10\% best males for test-day milk yields and 305-day milk yield, based on 305-day breeding value predicted by random regression analysis (MRA305)

\begin{tabular}{|c|c|c|c|c|c|c|c|c|c|c|c|c|}
\hline \multirow[t]{2}{*}{$\begin{array}{l}\text { Touro } \\
\text { Sire }\end{array}$} & \multirow[t]{2}{*}{ MRA305 } & \multirow[t]{2}{*}{ PTA305 } & \multicolumn{10}{|c|}{$\begin{array}{l}\text { Modelo de regressão aleatória (semana) } \\
\text { Random regression model (week) }\end{array}$} \\
\hline & & & 4 & 8 & 12 & 16 & 20 & 24 & 28 & 32 & 36 & 40 \\
\hline 89341 & 1 & 2 & 2 & 2 & 2 & 2 & 2 & 1 & 1 & 1 & 1 & 3 \\
\hline 84075 & 2 & 28 & 1 & 1 & 1 & 1 & 1 & 2 & 2 & 2 & 3 & 6 \\
\hline 85233 & 3 & 12 & 4 & 3 & 3 & 3 & 3 & 3 & 3 & 4 & 4 & 4 \\
\hline 78036 & 4 & 1 & 3 & 4 & 4 & 4 & 6 & 6 & 6 & 6 & 7 & 12 \\
\hline 84332 & 5 & 4 & 6 & 6 & 6 & 6 & 4 & 5 & 5 & 5 & 5 & 5 \\
\hline 72083 & 6 & 15 & 17 & 10 & 8 & 7 & 5 & 4 & 4 & 3 & 2 & 1 \\
\hline 88209 & 7 & 5 & 5 & 5 & 5 & 5 & 7 & 7 & 7 & 10 & 13 & 14 \\
\hline 86176 & 8 & 16 & 7 & 7 & 7 & 8 & 8 & 8 & 8 & 9 & 11 & 13 \\
\hline 77239 & 9 & 33 & 15 & 11 & 9 & 9 & 9 & 9 & 10 & 12 & 14 & 16 \\
\hline 86369 & 10 & 17 & 9 & 9 & 10 & 10 & 11 & 12 & 15 & 17 & 21 & 23 \\
\hline 71221 & 11 & 7 & 35 & 20 & 18 & 15 & 10 & 10 & 9 & 8 & 8 & 8 \\
\hline 72124 & 12 & 34 & 10 & 13 & 14 & 13 & 13 & 14 & 14 & 16 & 18 & 20 \\
\hline 78120 & 13 & 27 & 19 & 16 & 16 & 16 & 16 & 15 & 13 & 14 & 16 & 18 \\
\hline 65256 & 14 & 10 & 14 & 14 & 13 & 11 & 12 & 13 & 18 & 19 & 23 & 27 \\
\hline 83215 & 15 & 13 & 11 & 12 & 11 & 12 & 15 & 17 & 19 & 23 & 27 & 26 \\
\hline 85071 & 16 & 14 & 29 & 18 & 15 & 14 & 14 & 16 & 17 & 18 & 20 & 21 \\
\hline 72096 & 17 & 3 & 55 & 29 & 20 & 18 & 17 & 11 & 11 & 11 & 9 & 9 \\
\hline 83326 & 18 & 134 & 12 & 15 & 17 & 19 & 18 & 21 & 26 & 32 & 35 & 40 \\
\hline 70156 & 19 & 6 & 57 & 43 & 31 & 23 & 20 & 18 & 16 & 13 & 12 & 10 \\
\hline
\end{tabular}


preditos para estes períodos específicos da lactação (Kistemaker, 2003). Esse autor testou três medidas de persistência, que eram razões de valores genéticos em períodos parciais da lactação, usando MRA ajustados com polinômios de Legendre. Uma proposta para o Canadá, por exemplo, define a persistência por meio da subtração dos valores genéticos médios estimados no período entre 255 e 305 dias de lactação, pelos valores genéticos médios entre 50 e 70 dias de lactação, que deve ser o período aproximado em que ocorre o pico de lactação. Os MRA, por serem versáteis, permitem essas predições em qualquer fase da lactação.

No presente estudo, foram constatadas divergências na classificação dos animais quando os valores genéticos foram preditos pelo TDMO e MRA. Comparando PTA305 e MRA305, como critérios de seleção, foram constadas divergências na classificação dos touros, em ordem de classificação e em diferentes decas. Para as raças taurinas, essas divergências talvez fossem menores, quando comparadas às raças zebuínas ou nativas, pois as taurinas não apresentam problemas de persistência ou duração da lactação. Quanto às raças zebuínas ou nativas, existem poucas informações sobre o comportamento esperado de resultados obtidos pelo MRA e TDMO, quando os animais apresentam lactações curtas, e sobre os resultados obtidos por ambas as metodologias. No

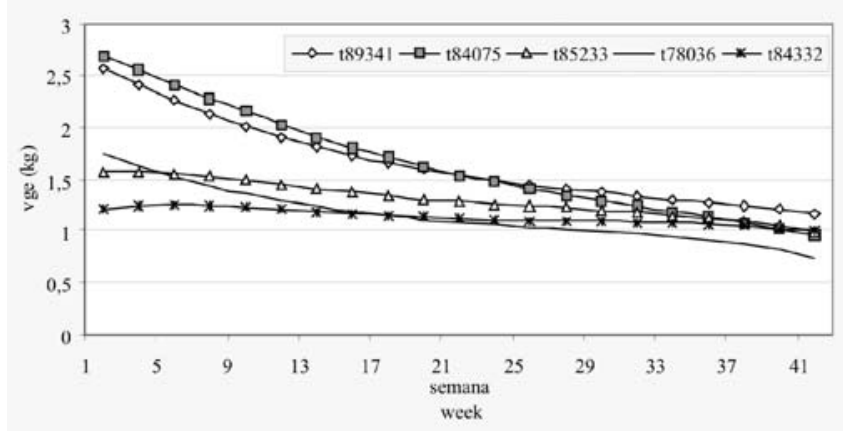

Figura 9 - Valores genéticos preditos para as produções de leite nas semanas de lactação pelo MRA para os cinco melhores touros, com base no valor genético predito para MRA305.

Figure 9 - Predicted breeding values for test-day milk yields by random regression of five best males based on MRA305. presente estudo, optou-se, como base para comparação, pela PTA305. Seriam necessários estudos sobre a simulação dos comportamentos das trajetórias dos animais para verificar os resultados esperados.

\section{Conclusões}

As correlações de ordem estimadas entre os valores genéticos indicaram que, para esse conjunto de dados, o "test-day model" ordinário foi inadequado para substituir a produção de leite acumulada até 305 dias nas avaliações genéticas de reprodutores.

A regressão aleatória foi uma metodologia mais adequada que o "test-day model" ordinário em programas de avaliação genética de bovinos leiteiros, visando substituir a produção acumulada até 305 dias, tradicionalmente usada.

Foi observada tendência dos modelos de regressão aleatória em favorecer a forma da curva de lactação, beneficiando os touros cuja queda nos valores genéticos após o pico da lactação foi menos acentuada.

A somatória dos valores genéticos preditos no período de 305 dias da lactação (MRA305) poderia ser adotada como um critério de seleção alternativo à PTA305, tradicionalmente empregada em avaliações genéticas.

Outros estudos, com conjuntos de dados mais representativos, devem ser conduzidos, visando comprovar as vantagens observadas no presente estudo em relação aos modelos de regressão aleatória.

\section{Literatura Citada}

BOLDMAN, K.G.; KRIESE, L.A.; VAN VLECK, L.D. et al. A manual for use of MTDFREML. USDA-ARS: Clay Center, Nebraska, USA. 1993. 120p.

BROTHERSTONE, S., WHITE, I.M.S., MEYER, K. Genetic modelling of daily yield using orthogonal polynomials and parametric curves. Journal of Animal Science, v.70, p.407-415, 2000.

EL FARO, L. Estimação de componentes de (co)variância para produção de leite no dia do controle de primeiras lactações de vacas caracu, aplicando-se "test-day models" de dimensão finita e modelos de regressão aleatória. Jaboticabal: Universidade Estadual Paulista, 2002. 120p. Tese (Doutorado em Zootecnia) - Universidade Estadual Paulista, 2002.

GADINI, C.H. Genetic evaluation of test day production traits and somatic cell score. Lincoln: University of Nebraska, 1997. 91p. Thesis (PHD) - University of Nebraska, 1997. 
JAMROZIK, J.; SCHAEFFER, L.R. Estimates of genetic parameters for a test day model with random regressions for yield traits of first lactation Holsteins. Journal of Dairy Science, v.80, p.762-770, 1997.

JAMROZIK, J.; KISTEMAKER, G.J.; DEKKERS, J.C.M. Comparison of possible covariates for use in a random regression model for analyses of test day yields. Journal of Dairy Science, v.80, p.2550-2556, 1997a.

JAMROZIK, J.; SCHAEFFER, L.R.; LIU, Z. et al. Multiple trait random regression test day model for production traits. In: INTERBULL MEETING, 16., 1997, Vienna, Austria. Proceedings...Vienna: 1997b. p.43-47.

KIRKPATRICK, M.; LOFSVOLD, D.; BULMER, M. Analysis of the inheritance, selection and evolution of growth trajectories. Genetics, v.124, p. 979-993. 1990.

KISTEMAKER, G.J. Comparison of persistency definitions in random regression test day models. In: INTERBULL TECHNICAL WORKSHOP, 30., 2003, Beltsville. Proceedings... Beltsville: 2003. Internet: http://wwwinterbull.slu.se.

MEYER, K. "DXMRR" - A program to estimate covariance functions for longitudinal data by REML. In: WORLD CONGRESS OF GENETICS APPLIED TO LIVESTOCK PRODUCTION, 6., 1998, Armidale, Australia. Proceedings... Armidale: University of New England (CD ROM). 1998a.

MEYER, K. Estimating covariance functions for longitudinal data using a random regression model. Genetic Selection Evolution, v. 30, p. 221-240. 1998b.

OLORI, V.E.; HILL, W.G.; BROTHERSTONE, S. The structure of the residual error variance of test day milk yield in random regression models. In: COMPUTATIONAL CATTLE BREEDING WORKSHOP, 1999, Finland. Proceedings... Tuusula, Finland, 1999a.
OLORI, V.E.; HILL, W.G.; MCGUIRK, B.J. et al. Estimating variance components for test day milk records by restricted maximum likelihood with a random regression animal model. Livestock Production Science, v.61, p.53-63, 1999 b.

PTAK, E.; SCHAEFFER, L.R. Use of test day yields for genetic evaluation of dairy sires and cows. Livestock Production Science, v.34, p.23-34, 1993.

STATATISCAL ANALYSES SYSTEM - SAS. User's guide: Statistics. Cary: 1992. 842p.

SCHAEFFER, L.R. Tópicos avançados em melhoramento animal: random regression models. Jaboticabal: Universidade Estadual Paulista, 1996. p.25-33. (notas do curso)

STRABEL, T.; MISZTAL, I. Genetic parameters for first and second lactation milk yields of Polish Black and White cattle with random regression test-day models. Journal of Dairy Science, v.82, p.2805-2810, 1999.

TIJANI, A.; WIGGANS, G.R.; Van TASSEL, C.P. et al. Use of (co)variance functions to describe (co)variances for test day yield. Journal of Dairy Science, v.82, 1999. Diposnível em: http://www.adsa.org/jds.

Van der WERF, J.H.J.; SCHAEFFER, L,R. Random Regression in animal breeding. (course notes). University of Guelph, Guelph, Canada. 58p. 1997.

Recebido em: 04/09/03

Aceito em: 29/11/04 